\title{
The Impacts of Cloud Computing Adoption at Higher Education Institutions: A SWOT Analysis
}

\author{
Mahmoud Odeh \\ Coventry University \\ Business school \\ Priory ST, Coventry, UK
}

\author{
Kevin Warwick \\ Coventry University \\ Priory ST, Coventry, UK
}

\author{
Alexeis Garcia-Perez \\ Coventry University \\ Business school \\ Priory ST, Coventry, UK
}

\begin{abstract}
The integration of advanced technologies within education has frequently enhanced teaching. In higher education it is not a surprise that using the latest developments in cloud computing improves learning practices and thus ensures they are more interactive, available, and convenient. The ease of integration, collaboration, and sharing of information and knowledge made possibleby cloud computing will be further enhanced if this technical advancement is used wisely and in a foolproof manner. In this paper, a SWOT analysis of the impact of cloud computing on higher education methodologies is presented. A SWOT analysis is here demonstrated to be a helpful guide in decision-making for all higher education institutions when considering the migration of their present learning systems to cloud based systems.
\end{abstract}

\section{General Terms}

Cloud computing at higher education institutions

\section{Keywords}

Cloud computing, e-learning, SWOT analysis, Web learning, higher education

\section{INTRODUCTION}

Cloud computing represents a recent leap in the provision of technological services. All the technical aspects that were once created locally for each and every business are provided as different services on a chargeable basis. This technological shift creates third party service providers, who deliver these services on a larger scale and remotely services which claimed of seeing more effective. Various services, such as the establishment and maintenance of software environments, storage, platforms, processing, are provided. Data that was previously kept under the consumer's own administration, in his or her secure domain, is extracted and relocated under the domain of the service provider.

Cloud computing services made available in the higher education arena promise to offer significant improvements in flexibility and increased agility for users, leading to greater value for money.

In any Internet-based learning system one of the most important issuesto address is the flexibility and convenience of using that system. With cloud computing, any educational organization can increase its technological infrastructure without having to compromiseon storage capacity and ad hoc computing requirements. This is because cloud computing, seen most simply as a group of high-end computers networked together, has the great potential to provide computation and storage resources as services. Therefore, educational institutions in both the public and private sectors can make use of these resources provided by cloud environments to extend and deliver state-of-the-art services to their own staff and students with fewer local resources and hardly any extra investment in IT infrastructure within the institution. With collaborative learning and knowledge-sharing as very desirable outcomes of cloud computing systems, several webbased universities have already secured their place, with multi-media and virtual classes on learning-based study materials readily available through their presence in the cloud $[23,36]$.

Moreover, the ubiquitous nature of cloud computing technology enables universities with cloud services to introduce their learning systems to students of developing countries where they do not have a physical presence. In developing countries in particular, e-learning via cloud computing could raise the level of education, literacy, and economic development where education is otherwise expensive, opportunities are limited, and economic disparities exist [8].

In this paper, a critical assessment of the technical impact of using cloud computing in higher education is carried out via a SWOT analysis.

\subsection{Background - Previous research}

Cloud computing is a subscription-based service where users can obtain different types of services: storage space, processing, and networking resources. The cloud makes it possible to access clients 'data from anywhere at any time. While a traditional computer setup requires the same location for both the users and the data storage device, the cloud removes that necessity. Cloud computing technologies eliminate the need to have a physical location for the purpose of data storage [29].

McDonald et al. [28] have performed an in-depth analysis and elaboration regarding the implementation of the cloud environment in higher education institutions. The objectives of their research were: to revise the values of the environmental costs and benefits of cloud computing; to investigate different environmental impacts that cloud computing has for institutional activities which are not research; to propose changes within institutional governance and advocate procedures which would enforce the use of cloud computing, and to make recommendations to the Joint Information Systems Committee for further improvements. They analyzed the use of cloud computing in UK higher education institutions in the period 2008/09. This analyses demonstrated that the institutions had very moderate usage of cloud services in place (basically student mail-boxes), but that the institutions had clear intentions to improve that state and to replace the existing IT infrastructure with cloud-based solutions. 
McDonald et al. [28] showed that this approach would bring a significant number of benefits to the institutions (conserving energy, saving on man-power and maintenance). They developed 4 near-future scenarios for the use of cloud computing in higher education: Cloud Workspace, LargeScale Cloud Storage, Cloud-Enabled Learning (both virtual and personal) and the Academic Cloud Scenario. Their recommendations were given to the JISC in order to accelerate the process of cloud adoption in HE institutions.

Hussein et al. [37] give an original description of the migration of an IT system from a company's in-house datacentre to Amazon's cloud solution EC2. They analyzed the migration of a UK-based corporation that offers IT services to the oil and gas industry. The financial and technical issues were subject to analysis, and the data obtained can serve as guidance for other similar cases. The study identified the benefits and drawbacks associated with the migration and its impact upon the company's entire staff body. The research described all the steps required for the migration process (cost analysis, identifying stakeholders, impact analysis, and finally, the database migration). After successful migration, the following benefits were identified: reduced costs, the ability to manage income and costs, the ability to offer new services, a chance for the company's growth, avoidance of repetitive work, etc. The following drawbacks (risks) were also identified: dependency on a third party, problems with the acceptance of the new technology, partial lay-off of staff, decreased satisfaction, security concerns etc.

The adoption of cloud services in higher education institutions is elaborated in the book "Tower and the Cloud", edited by Katz [40]. Different authors elaborate on several topics, such as: higher education and Information Technology; globalization of higher education; IT governance; open information, open source, open content; scholarship in the cloudy world. The authors concluded that today's classroom education has been augmented and replaced by online learning. In the future, the lines between institutions and organizations will fade, as will the lines between consumers and service-providers, and between learners and instructors. Moreover, new ways of knowledge, more creative platforms for learning, and better ways of understanding the new technology will create new opportunities for all active participants in the educational process.

Jensen et al. [41] investigated different security threats: cloud malware injection, metadata spoofing attacks, flooding attacks, direct and indirect denial of service (DOS), etc. For the cloud malware attack, the attacker will first create its own service on the Cloud (e.g. SaaS, or PaaS). The attacker then maliciously attempts to convince the cloud provider that the new service is a valid offering for a given organization. When the legitimate users ask for particular cloud services, they will be re-directed towards the fake ones. A possible defense against this attack is to check the integrity of the service prior to its launch and distribution to its final intended users. The metadata spoofing attacks are based on web-service malware commands. If the Cloud system consists of a WSDL repository, changing the service invocation commands will result in wrong (harmful) SOAP messages. The authors concluded that although these types of security threats are serious, careful planning can lead to avoiding them.

The issue of privacy has been taken into account in the reseach of Pearson [45]. In today's world, the user's privacy is continually under attack, and users' personal data is permanently hampered by large marketing vendors. Different types of information should be protected (hidden) from un- authorized use, such as personal data, corporate data, sensitive data, digital content, usage data, software configuration data. In cloud computing, several privacy risks can occur: leakage of personal data, disruption of corporate policies and procedures, liability and credibility concerns for the cloud providers, non-compliance and function creep for the application providers etc. Pearson states that privacy is a very important issue in the design of cloud services, and he suggests several measures to ensure privacy protection as follows: transparency- the cloud provider has to inform the users which data will be collected; choice - the user should decide by himself whether to provide his data or not; purpose - the data should be used only for the specific purpose for which it is collected; auditing - the cloud provider has to appoint a dedicated person to take care of the user's data and privacy issues.

Finally, Hosseini et al. [37] emphasize different types of risks to which the cloud is open:

Deterioration of customer care \& service quality.

Decrease in satisfaction.

Departmental downsizing.

Uncertainty about new technology.

Lack of supporting resources.

In summary, their results illustrate that while financial and technological issues are certainly important, organizational aspects should also be considered. These should be considered especially from the point of view of service quality and customer care, while also taking into account organizational structure and the implications of being heavily dependent on a third party for providing customers with a product or service.

Cloud computing could be viewed as a cheap and convenient option for processing large amounts of information in schools and universities. According to Wang and Xing [17], the extensive use of cloud computing applications in education informatization will help to provide solutions for the distribution of educational resources, and increase the ability to share information.

A four-stage process of strategies for the adoption of cloud computing in higher education has been suggestedby Meanwhile Mousad and Huang [11]. These four stages are 1) Developing knowledge, feasibility, and an initial plan for cloud computing, 2) Evaluating the current system and experimenting with how the system changes with the use of the cloud, 3) Choosing the correct cloud computing solution, and 4) Implementation and management of a solution.Each and every stage should be performed with different strategies to make the migration or the transition easy and less prone to error.

\section{WHAT IS SWOT ANALYSIS?}

SWOT analysis is a technique for understandinga process or project's strengths and weaknesses, and for identifying both the opportunities and possible threats that it may face [13]. It involves specifying the objectives of the project and identifying the internal and external factors that are favourable and unfavourable to these objectives. The SWOT analysis is a very powerful tool, since the findings of this analysis can be used to understand the advantages of any project, and can therefore help to uncover the various opportunities that can be exploited. 
By understanding the weaknesses of a project, the various threats that may be faced in the future are highlighted, and thus a better strategy can be tailor-made to manage and eliminate these threats. By using a SWOT analysis, any organization can develop a strategy to take the business forward, with the potential to surpass its competitors in the market.

Before presenting a detailed SWOT analysis of the technical impact of adopting cloud computing in higher education, we briefly outline here the various computing segments that are taken into account when considering a migration to cloud computing.
- Application Segment: This is the segment where the front-end of any application is installed and accessed in the cloud.

- Storage: This segment provides the storage infrastructure where huge databases are involved.

- Connectivity: Connectivity is the segment which provides the interconnection or networking, making high speed data transmission possible.

According to our analysis, the various strengths, weaknesses, opportunities, and threats involved can be viewed as shown in the table below.

Table 1. Cloud computing SWOT analysis

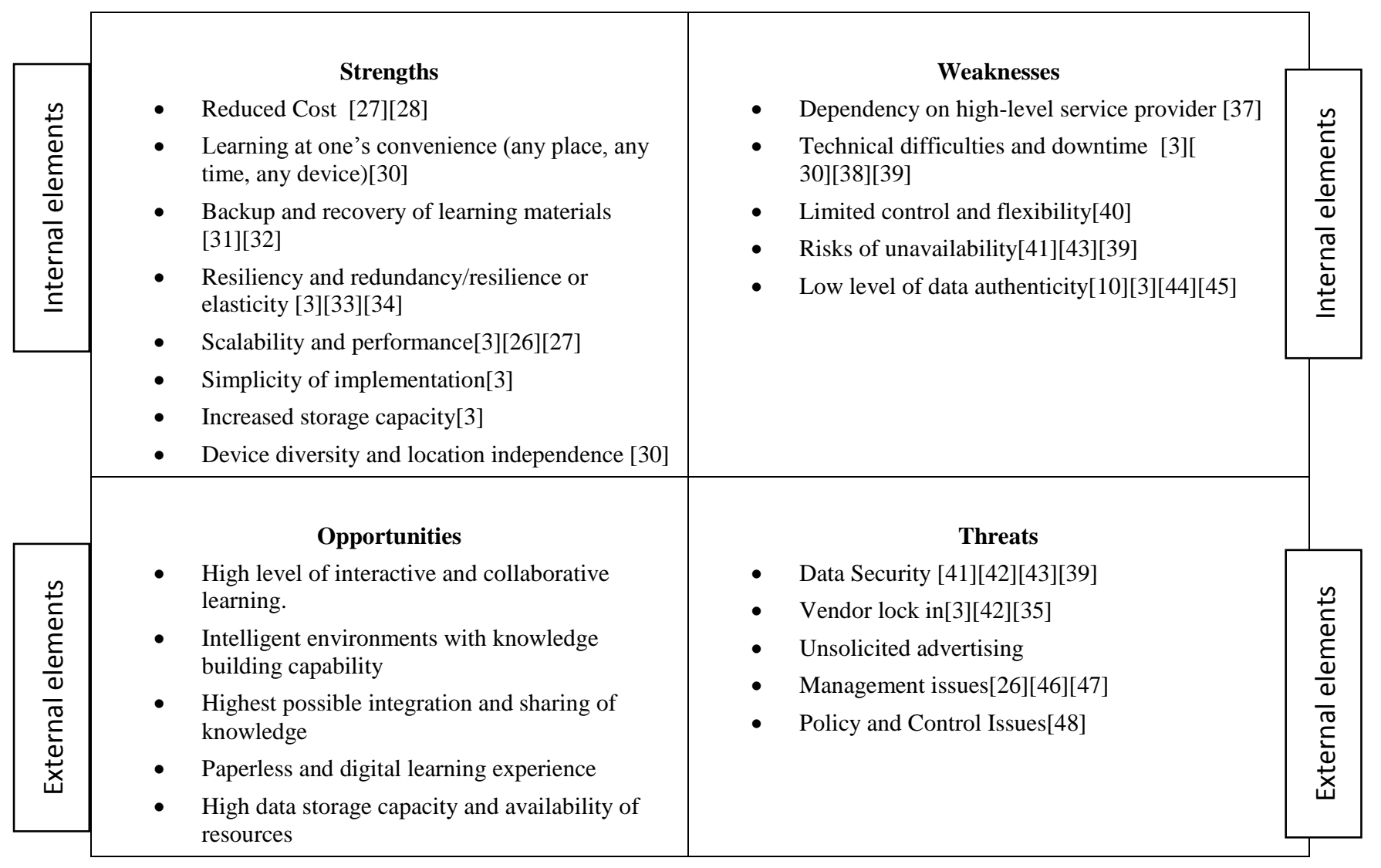

\subsection{Strengths}

In SWOT analysis, strengths are those internal characteristics that will be beneficial for the project. Adopting Cloud computing in higher education does not really need any costly IT infrastructure or software enviroment. Cloud computing by itself offers all these services either on a "pay-as-you-go" or 'rent-by-the-hour" system, as introduced by Amazon's Elastic Cloud Computing (EC2), which claims to provide "resizable computer capacity in the cloud" [4].

According to a 2011 survey by the Pew Research Center, " $75 \%$ of adults say college is too expensive for most Americans to afford. Moreover, 57\% said that the higher education system in the U.S. fails to provide students with good value for the money they and their families spend." [6].

EC2 basically rents out to research scholars computing resources and/or software environments, which otherwise can be extremely expensive if much investment is made in in- house servers. The Electrical Engineering and Computer Science department at the University of California, Berkeley, with its concept of Virtual Computing Labs offered to its students, has benefited substantially from a much reduced cost as well as avoiding the twin-damage of over provisioning and under provisioning of internal data centers [3].

Setting up a learning system in the cloud gives the flexibility for a learner or a faculty member to use the learning portal at their convenience. Also since the portal is hosted in the cloud, it is always available through the internet, and hence any remotely located user can access materials and use them to learn via the internet. In a cloud-based learning system, the storage infrastucture is provided as a service on a pay-per-use basis. These storage systems are highly advanced and have multiple mirroring of data. Data backup servers are equipped to take backups automatically after specific periods. With all these facilities, higher education systems that adopt cloud 
computing will have improved data backup and recovery mechanisms.

The implementation of elastic storage with Amazon S3 at the Universities of Stanford and Texas is another example of the success of using cloud services where location independence and high end storage facilities are offered by different providers. This implementation has improved storage capacity and the independence of access of the computing environment, yet at the same time has lowered the cost [1].

Resilience is another strength of cloud-based learning systems $[19,20]$. By resilient systems we mean here that in any cloud service there is always a form of failover that distributes redundant implementations of IT resources across physical locations. Resilience is made possible by keeping more than one similar resource. In this way greater redundancy of resources can make the service more resilient.

Elasticity in cloud computing is a characteristic in cloud technology that can handle the increasing of workload by increasing resources amount through providing new virtual resources dynamically. It is automated ability of cloudbacked systems to transparently scale the various IT resources, as required during runtime conditions [49]. Whereas, Scalability is to enlarge and increase the resources amount in advance by expect the maximum system future needs [49]. Cloud-based learning systems offer high scalability and Elasticity feature. Hence, Cloud computing technology provides high performance and massive resources.

Another strength of Cloud computing that the deployment of cloud services is easy since these services do not normally need any high level installations or configurations at the client's site. All the services can be connected directly to the cloud service. The diversity of devices that can be used by Cloud computing is another main strength. Most portable media and hand-held devices including smart phones, Tablets, laptops PDAs and ebook readers can access the cloud-based portal for learning purposes from any location since the portal is hosted in the cloud $[9,50]$.

\subsection{Weaknesses}

From the SWOT analysis point of view, weaknesses are those internal characteristics of the project that may become disadvantageous compared to other projects. There are several weaknesses that should not be ignored while specifically adopting cloud computing in higher education.

One of the major weaknesses of cloud-based learning systems is the high dependency of the tenant on the service provider [14]. Since the entire application, the storage and the environment itself is hosted in the cloud, there is practically nothing the users are in control of. Everything is dependant on the cloud service provider's network.

Other weaknesses are due to the technical difficulties that appear in the form of bridging the gap between the traditionally run and managed application when migrating to a highly advanced cloud computing service. The application, databases and the connectivities could however be fine-tuned to the Processor in order to avoid some if not all of these difficulties.

Trouble-shooting an application which is hosted in a service provider's server is also very difficult. Cloud-based services, like learning systems cannot be fully controlled at the tenant level. This is because, apart from a very few administrative controls over the application and the database hosted in the cloud, the service provider may not be interested in giving any other controls over his service. Hence the amount of flexibility and control users get while hosted in their own server is lacking.

Other than this, there can be risks of unavailability of various resources in a cloud-based system. This is because many applications would most likely be hosted in a cloud-based system and there is the chance that all the resources are being used up or engaged at a particular time. Moreover, some of the servers might become over-loaded. Authenticity of the data obtained from a cloud service is another topic of concern $[16,10]$. Data could be corrupted due to security breaches in the cloud network. Also, since there are multiple tenants that have hosted their applications and their data in a cloud, so it is possible that the data that is retrieved may in fact be from acompletely diiferent data base. Proper and effictive security check should be employed in order to avoid this type of glitches.

\subsection{Opportunities}

Opportunities are those external elements that a project can use for its advantage and success. One of the major opportunities that can be explored and tapped into a cloudbased higher education system, is the level of interaction and collaborative learning.

Interactive learning systems may provide a one-to-one teacher-student environment to the users. Collaborative learning is enabled by giving the users access to the vast amount of learning material and media over the portal, which makes the learning experience richer. Oracle APEX is a collaborative learning environment used to process Webbased SQL requests, and offers a platform for quick, and highly reliable, development and web applications [5].

The cloud also provides intelligent environments which allocate various resources elastically at run time. In cloudbased higher education systems, knowledge accumulation is done in a redundant and distributed manner. This enables the system to switch over to different resources as and when a particular server resource becomes unavailable. This functionality of resilience of a cloud-based system gives the project an opportunity to be an intelligent system with in-built knowledge-building capabilities [24].

The most important feature that should be used as an opportunity for the adoption of cloud computing is its ease of integration. The various learning resources such as multimedia-based study material and other interactive media, in various stuctures and formats, are integrated and made available to the users. This is because the cloud services usually support all formats and structures and have an efficient storage structure to use on them.

Sharing of knowledge is also made possible by interactive sessions and cloud-based forums. The core point is that a project eliminates the huge amount of printed study material that would otherwise be supplied to students on a higher education course, thereby reducing the carbon footprint.

Google Sites is a leader in teaching applications, and presently much teaching practice and research supported by cloud computing [25]. By keeping all the material digitized and ensuring its high availability over the network, it provides users a highly flexible digital learning experience.

Cloud-based services have a very efficient storage system with multiple servers of high storage capacity with mirroring and regular-backup capabilities. Storage as a service ensures that the same data is stored in redundant resources, even 
though one resource may be unavailable, data can be retrieved from another resource. This may ensures a high level of availability in e-learning resources [15].

\subsection{Threats}

In SWOT analysis, threats are those external elements that, unless minimized, can cause the project to fail [13]. The security of data in a cloud-based service is particular concern. This is because of the lack of control by the service provider and because of the lack of information about the type of securitythe service provider employs.

Various threats, such as Man-in-the-middle and DoS (Denial of Service) can apply to both data-in-transit and also data that is stored [7]. Distributed Denial of Service (DDoS) attacks, in which many nodes systems all attack one single node at the same time with a flood of messages, may cause the whole system to go down.

Lock-in or vendor lock-in, is a technique which ensures that the customer or tenant that wants to use a cloud service must also use a particular third party vendor service. Some of the cloud service providers even impose a restriction on the type of software; application; and hardware to be employed in order to make use of their services. Moreover, these types of services also carry heavy switching costs between competing vendors. For example, Google uses BigTable for storage where Facebook uses Cassandra and Amazon uses Dynamo [12]. Since there is no common interface between these databases, migration between them is either not possible or is quite costly.

Unsolicited advertising or spamming is another considerable threat that is apparent in many cloud-based systems. ANDROIDOS_SNDAPPS.SM is one such adware found in application store. This adware penetrates a consumer device and displays unsolicited advertisements. During an execution process, it gathers specific information from a user's device. It then sends this information to a website [2]. This particular junk mail can jam the communications lines which causing higher down times and seriously deteriorate the availability of resources.

Even a data storage service can be misused by this kind of threat. These should be avoided by ensuring that the service provider has proper anti-spamming methodologies in place. However, most cloud service providers lack a sense of visibility in managing the cloud. They also find it very difficult to manage multi-tenancy. Since many companies as tenants are running virtual instances of their applications completely independently of each other, the management of these as well as the entire infrastructure is very complex.

The access control and authentication policy for a specific cloud service should be done in a very professional way. Since there is always a lack of control for the tenant, the type of controls and the extent of control that is to be given to a tenant should be confirmed in the software level agreement (SLA) in order to avoid further problems [21,22].

\section{CONCLUSIONS}

Cloud computing, while it has numerous benefits for higher education institutions, also has some critical limitations or constraints. These constraints can be of either a technical or managerial nature. The evidence suggests that cloud computing will facilitate more learning experiences for students by increasing access to information and enabling collaboration, correlation, and data sharing. Therefore, it is likely that it will become a more pervasive component of the technology infrastructure in education institutions. However, legal and policy constraints should be taken very seriously in the transition period when migrating to the cloud. The success of any cloud-based higher education system also depends on the selected type of cloud deployment model. A suggestion for this is for universities to concentrate on private cloud deployments in order to enjoy cloud-based facilities with minimum risks.

\section{REFERENCES}

[1] Amazon Web Services (AWS) (18/11/2014). - Cloud Computing Services. Available: http://aws.amazon.com/education/customerexperiences/\#6.

[2] ANDROIDOS_SNDAPPS.SM (18/11/2014). Available: http://www.trendmicro.com/vinfo/us/threatencyclopedia/malware/ANDROIDOS_SNDAPPS.SM.

[3] A. Fox, R. Griffith, A. Joseph, R. Katz, A. Konwinski, G. Lee, et al., "Above the clouds: A Berkeley view of cloud computing," Dept. Electrical Eng. and Comput. Sciences, University of California, Berkeley, Rep. UCB/EECS, vol. 28, p. 13, 2009.

[4] AWS | Amazon Elastic (18/11/2014). Compute Cloud (EC2) - Scalable Cloud Hosting. Available: http://aws.amazon.com/ec2/.

[5] D. Baker \& T.Jennings. Oracle Database 2 Day + Application Express Developer's Guide, Release 4.0. E15516- 04. Oracle, 2010. Retrieved from https://docs.oracle.com/cd/E17556_01/appdev.40/e15516 .pdf.

[6] College Graduation: Weighing the Cost ? and the Payoff | Pew Research Center. (n.d.). Retrieved from http://www.pewresearch.org/2012/05/17/collegegraduation-weighing-the-cost-and-the-payoff/.

[7] D. Jamil and H. Zaki, "Cloud computing security," International Journal of Engineering Science and Technology, vol. 3, pp. 3478-3483, 2011.

[8] S. Okai, M. Uddin, A. Arshad, R. Alsaqour, and A. Shah, "Cloud Computing Adoption Model for Universities to Increase ICT Proficiency," SAGE Open, vol. 4, p. 2158244014546461, 2014

[9] B. Furht and A. Escalante, Handbook of Cloud Computing: Springer US, 2010.

[10] D. Zissis and D. Lekkas, "Addressing cloud computing security issues," Future Generation computer systems, vol. 28, pp. 583-592, 2012.

[11] M. Masud, X. Huang, and J. Yong, "Cloud computing for higher education: a roadmap," in Computer Supported Cooperative Work in Design (CSCWD), 2012 IEEE 16th International Conference on, ed., 2012, pp. 552-557.

[12] P. Hofmann and D. Woods, "Cloud computing: the limits of public clouds for business applications," Internet Computing, IEEE, vol. 14, pp. 90-93, 2010. 
[13] T. Hill and R. Westbrook, "SWOT analysis: it's time for a product recall," Long range planning, vol. 30, pp. 46-52, 1997.

[14] L. Grandinetti, Pervasive Cloud Computing Technologies: Future Outlooks and Interdisciplinary Perspectives: Future Outlooks and Interdisciplinary Perspectives: IGI Global, 2013.

[15] D. Madan, A. Pant, S. Kumar, and A. Arora, "E-learning based on Cloud Computing," International Journal of Advanced Research in Computer Science and Software Engineering, vol. 2, 2012.

[16] T. Zia, A. Zomaya, V. Varadharajan, and M. Mao, Security and Privacy in Communication Networks: 9th International ICST Conference, SecureComm 2013, Revised Selected Papers: Springer Publishing Company, Incorporated, 2014.

[17] Bo Wang; HongYu Xing, "The application of cloud computing in education informatization", International Conference on Computer Science and Service System, Nanjing, pp.2673-2676, 27-29 June 2011.

[18] M. Pandya, "Cloud computing for libraries: A SWOT analysis," 2012.

[19] P. Verissimo, A. Bessani, and M. Pasin, "The TClouds architecture: Open and resilient cloud-of-clouds computing," in Dependable Systems and Networks Workshops (DSN-W), 2012 IEEE/IFIP 42nd International Conference on, 2012, pp. 1-6.

[20] R. Jhawar and V. Piuri, "Fault tolerance and resilience in cloud computing environments," Computer and information security handbook, 2nd edn. Morgan Kaufmann, Burlington, 2013.

[21] R. Buyya, C. S. Yeo, S. Venugopal, J. Broberg, and I. Brandic, "Cloud computing and emerging IT platforms: Vision, hype, and reality for delivering computing as the 5th utility," Future Generation computer systems, vol. 25 , pp. 599-616, 2009.

[22] L. Wu, S. K. Garg, and R. Buyya, "SLA-based resource allocation for software as a service provider (SaaS) in cloud computing environments," in Cluster, Cloud and Grid Computing (CCGrid), 2011 11th IEEE/ACM International Symposium on, 2011, pp. 195-204.

[23] M. A. H. Masud and X. Huang, "An e-learning system architecture based on cloud computing," system, vol. 10, 2012.

[24] I. M. Abbadi and A. Martin, "Trust in the Cloud," information security technical report, vol. 16, pp. 108$114,2011$.

[25] G. Attwell, "Personal Learning Environments-the future of eLearning?," eLearning papers, vol. 2, pp. 1-8, 2007.

[26] J. Dibbern, T. Goles, R. Hirschheim, and B. Jayatilaka, "Information systems outsourcing: a survey and analysis of the literature," ACM SIGMIS Database, vol. 35, no. 4, pp. 6-102, Nov.2004.
[27] L. Herbert and J. Erickson, "The ROI Of Software-As-AService," Forrester Research, 2009.

[28] D. McDonald, A. MacDonald, and C. Breslin, "Final report from the JISC review of the environmental and organisational implications of cloud computing in higher and further education," University of Strathclyde and JISC, 2010

[29] A. Huth and J. Cebula, "The basics of cloud computing," United States Computer, 2011.

[30] J. F. Rayport and A. Heyward, "Envisioning the Cloud: The Next Computing Paradigm,"Marketspace, 2009.

[31] J. Varia, "Architecting for the Cloud: Best Practices," Amazon Web Services, 2010.

[32] T. Wood, E. Cecchet, K. Ramakrishnan, P. Shenoy, J. Van Der Merwe, and A. Venkataramani, "Disaster recovery as a cloud service: Economic benefits \& deployment challenges," in 2nd USENIX Workshop on Hot Topics in Cloud Computing, 2010, pp. 1-7.

[33] S. C. Misra and A. Mondal, "Identification of a company's suitability for the adoption of cloud computing and modelling its corresponding Return on Investment," Mathematical and Computer Modelling, Mar. 2010.

[34] B. Suleiman, S. Sakr, R. Jeffery, and A. Liu, "On understanding the economics and elasticity challenges of deploying business applications on public cloud infrastructure," Journal of Internet Services and Applications, vol. 2, no. 3, pp. 1-21, Dec. 2011.

[35] J. Jurison, "The role of risk and return in information technology outsourcing decisions,"Journal of Information Technology, vol. 10, no. 4, pp. 239-247, Dec. 1995.

[36] A. Fernandez, D. Peralta, F. Herrera, and J. Benítez, "An overview of e-learning in cloud computing," in Workshop on Learning Technology for Education in Cloud (LTEC'12), 2012, pp. 35-46.

[37] A. Khajeh-hosseini, D. Greenwood, and I. Sommerville, "Cloud Migration: A Case Study of Migrating an Enterprise IT System to IaaS," in IEEE 3rd Int. Conf. on Cloud Computing, 201, pp. 450-457.

[38] K. Sripanidkulchai, S. Sahu, Y. Ruan, A. Shaikh, and C. Dorai, "Are clouds ready for large distributed applications?," in ACM SIGOPS Operating Systems Review, 2010, vol. 44, no. 2, pp. 18-23.

[39] R. Clarke, "Computing Clouds on the Horizon?Benefits and Risks from the User's Perspective," in 23rd Bled eConference, 2010.

[40] R. Yanosky, "From Users to Choosers: The Cloud and the Changing Shape of Enterprise Authority," in The Tower and the Cloud, R. Katz, Ed. EDUCAUSE, 2008, pp. 126-136.

[41] M. Jensen, J. O. Schwenk, N. Gruschka, and L. L. Iacono, "On Technical Security Issues in Cloud 
Computing," in IEEE International Conference on Cloud Computing (CLOUD-II 2009), 2009, pp. 109-116.

[42] D. Catteddu and G. Hogben, "Cloud Computing: benefits, risks and recommendations for information security," European Network and Information Security Agency (ENISA), 2009.

[43] T. Ristenpart, E. Tromer, H. Shacham, and S. Savage, "Hey, you, get off of my cloud: exploring information leakage in third-party compute clouds," in 16th ACM Conference on Computer and Communications Security, 2009, pp. 199-212.

[44] J. Viega, "Cloud Computing and the Common Man," Computer, vol. 42, no. 8, pp. 106-108, 2009.

[45] S. Pearson, "Taking account of privacy when designing cloud computing services," in ICSE Workshop on Software Engineering Challenges of Cloud Computing, 2009, pp. 44-52.
[46] B. A. Aubert, M. Patry, and S. Rivard, "A framework for information technology outsourcing risk management," ACM SIGMIS Database, vol. 36, no. 4, pp. 9-28, Oct. 2005.

[47] M. Earl, “The risks of outsourcing IT," Sloan Management Review, pp. 26-32, 1996.

[48] B. Grobauer and T. Schreck, "Towards incident handling in the cloud," in Proceedings of the 2010 ACM workshop on Cloud computing security workshop (CCSW '10), 2010, pp. $77-85$.

[49] P. Sobeslavsky, T. Sardes, I. Rhône-Alpes, N. De Palma, F. Boyer, and B. Dillenseger, "Elasticity in cloud computing," Master's thesis, Joseph Fourier University, ENSIMAG, Grenoble, France, 2011. [50] Q. Zhang, L. Cheng, and R. Boutaba, "Cloud computing: state-of-theart and research challenges," Journal of internet services and applications, vol. 1, pp. 7-18, 2010. 